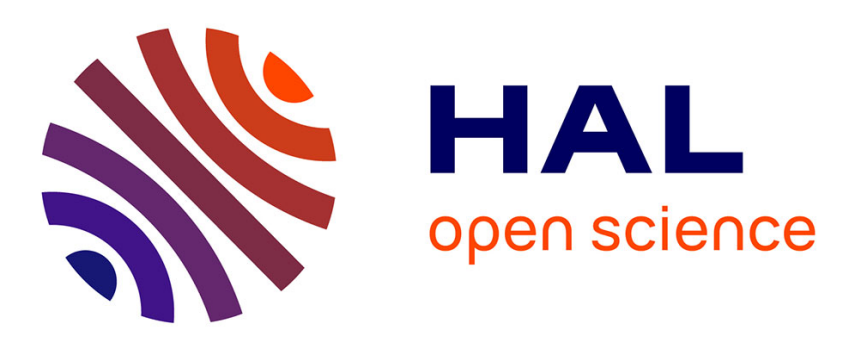

\title{
High Frequency Demodulation Technique for Instantaneous Angular Speed Estimation
}

Frédéric Bonnardot, Khalid Lizoul, Saad Errafik, Hugo André, François

Guillet

\section{- To cite this version:}

Frédéric Bonnardot, Khalid Lizoul, Saad Errafik, Hugo André, François Guillet. High Frequency Demodulation Technique for Instantaneous Angular Speed Estimation. Surveillance, Vishno and AVE conferences, INSA-Lyon, Université de Lyon, Jul 2019, Lyon, France. hal-02188565

\section{HAL Id: hal-02188565 \\ https://hal.science/hal-02188565}

Submitted on 18 Jul 2019

HAL is a multi-disciplinary open access archive for the deposit and dissemination of scientific research documents, whether they are published or not. The documents may come from teaching and research institutions in France or abroad, or from public or private research centers.
L'archive ouverte pluridisciplinaire HAL, est destinée au dépôt et à la diffusion de documents scientifiques de niveau recherche, publiés ou non, émanant des établissements d'enseignement et de recherche français ou étrangers, des laboratoires publics ou privés. 


\title{
High Frequency Demodulation Technique for Instantaneous Angular Speed Estimation
}

\author{
Frédéric Bonnardot ${ }^{1}$, Khalid Lizoul ${ }^{1}$, Saad Errafik ${ }^{1}$, Hugo André ${ }^{1}$, François Guillet ${ }^{1}$ \\ ${ }^{1}$ Univ Lyon, UJM Saint Etienne, LASPI, EA 3059 \\ F-42334, IUT de Roanne, France. \\ \{frederic.bonnardot\}@univ-st-etienne.fr
}

\begin{abstract}
This paper adapts the super-heterodyne method from telecommunication field to speed estimation based on optical encoder signal.

This method uses an analog frequency shifting before sampling of the signal. Therefore, the required sampling frequency is reduced and linked with the speed fluctuation frequency. Spectrum of optical encoder signal is analysed and used to explain how to set up the frequency shift and limitations. Finally, a comparison is made with elapsed time.
\end{abstract}

\section{Introduction}

Many vibration monitoring methods use an accelerometer sensor in order to diagnose the systems. Instantaneous Angular Speed (IAS) has appeared to provide a new source of information on the rotating machine and it is commonly used to diagnose faults such as bearing faults[1], gearboxes [2], $\ldots$

Classically, IAS use an optical encoder as a speed sensor. This sensor is available in many rotating machines (especially for speed and position control). It provides a square / sine wave with a frequency proportional to the rotation speed. Thus, this signal is frequency modulated by the system speed variations. There are two major methods for recording IAS signal: timer / counter technic [3], and ADC- based methods [4]. The latter is limited by the capability of the ADC-board to collect the data (sampling frequency) which could restrict the use of higher encoder resolution and introduces spatial aliasing.

In this study, we are interested in IAS estimation using a super-heterodyne like demodulation technique. The idea is to be able to acquire the speed signal with relatively higher resolution without using a higher sampling frequency. The IAS signal is first analogously shifted in frequency domain in order to be acquired at a lower sampling rate. Then, the sampled signal is further treated to get IAS.

A first part presents the measurement principle, a second part discuss about limitations, finally a last part show a comparison with elapsed time technique.

\section{Measurement principle}

In frequency demodulation technique, the signal is recorded at a high sample rate in order to exploit only the spectral content between $\left[f_{\text {cod }}-B / 2 ; f_{\text {cod }}+B / 2\right]$. The idea of super-heterodyne technique illustrated by figure 1 that come from telecommunication is to shift analogically the optical encoder signal from the band $\left[f_{\text {cod }}-B / 2 ; f_{\text {cod }}+B / 2\right]$ to the band $[\varepsilon ; \varepsilon+B]$ by using an analog multiplier $(\varepsilon$ is a small margin). Therefore, the recorded signal could be recorded with a sampling frequency greater than $2 \times B$. Since the band $B$ (a few kiloHertz) is smaller than optical encoder frequency, it becomes possible to use high resolution (4096 lines per revolution) with a classical data acquisition device. The demodulation effect is simply compensated after computing instantaneous speed by adding a constant frequency. 


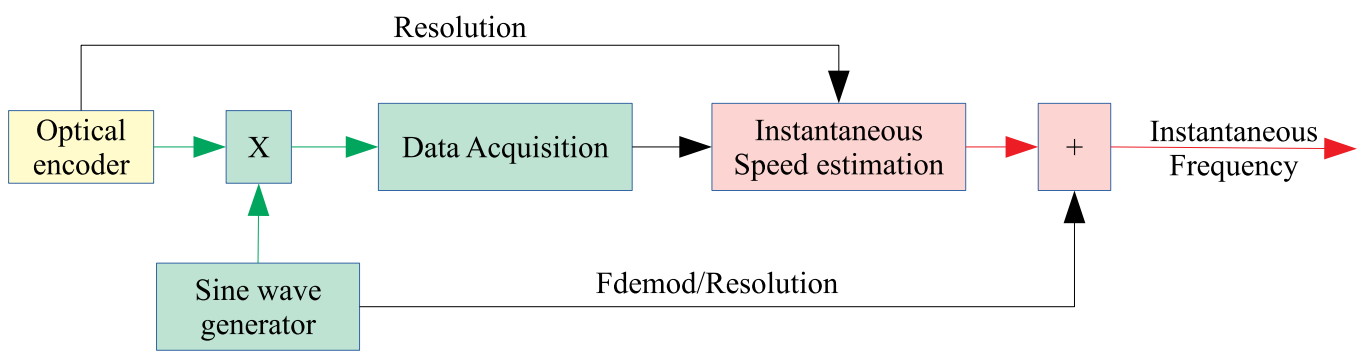

Figure 1: Principle of High frequency Demodulation Technique.

In order to illustrates the analog frequency shifting part, the sine wave generator, optical encoder signal and product signal has been acquired. Due to the limitation of data acquisition device, an optical encoder resolution of 256 lines has been used (to visualise original optical encoder signal). The sine wave generator has been set to $F_{m o d}=4 \mathrm{kHz}$. The spectrum of each signal is presented in figure 2 .

The first spectrum is the spectrum of the sine wave generator. On top of the peak at $4 \mathrm{kHz}$, a third harmonic $68 d B$ lower (i.e. amplitude 2400 lower) indicate the quality of the generator. It will also ask the question latter of the influence of a third harmonic on low-cost generators. The low noise in the spectrum is produced by the engine inverter as well as the generator.

The second spectrum corresponds to the optical encoder and was already studied in the first part. The effect of multiplication of encoder signal by sine wave results in a displacement of each component of the encoder spectrum by $F_{\text {mod }}$ on the left and on the right as indicated by the arrow. Each component is identified by a colour to help analysis after demodulation.

The last spectrum shows the demodulated signals by using the analog multiplier. Each optical encoder component has been shifted in both left and right direction. The amplitude ratio of $20 d B$ between $1^{\text {st }}$ and $3^{\text {rd }}$ optical encoder harmonic was kept after demodulation (on the bottom spectrum).

Unfortunately, components at $f_{\text {cod }}, 3 \cdot f_{\text {cod }}$ (marked in gray) and $F_{\text {mod }}$ are present after demodulation. It means that the product $p(t)$ signal is:

$$
\begin{aligned}
p(t) & =\left[\text { encoder }(t)+\varepsilon_{e}\right] \cdot\left[\sin \left(2 \pi F_{\text {mod }} t\right)+\varepsilon_{s}\right] \\
& =\text { encoder }(t) \cdot \sin \left(2 \pi F_{\text {mod }} t\right)+\varepsilon_{e} \cdot \sin \left(2 \pi F_{\text {mod }} t\right)+\varepsilon_{s} \cdot \operatorname{encoder}(t)
\end{aligned}
$$

Where :

- encoder $(t)$ is a zero mean version of encoder signal obtained by a high pass filter,

- $\varepsilon_{e}$ is an DC component (introduced by electronic),

- $\varepsilon_{s}$ is an DC component (introduced by electronic despite the fine tune of the sine wave generator).

The attenuation between the gray signal at $f_{\text {cod }}$ and the original red signal is $41 d B$, it means that $\varepsilon_{s}=0.009$. By looking at the sine wave the value $\varepsilon_{e}=0.001$ could be identified.

The values $\varepsilon_{e}$ and $\varepsilon_{s}$ are in fact small, and, reduce them necessitate higher precision offset adjustment.

In practice the last spectrum corresponds to the only acquired signal. The only interesting part of the signal is the left red pattern corresponding to the demodulated $f_{\text {cod }}$ part. This part could be acquired by an acquisition with a sampling frequency of $8 \mathrm{kHz}$.

After acquisition, a classical instantaneous frequency scheme is applied (band pass filtering, analytic signal extraction, differentiation). A simple addition of $F_{m o d} / N_{p p t}$ is required to compensate the frequency shift of $F_{\text {mod }}$.

It should be noted that in real application the optical encoder frequency $F_{\text {cod }}$ should be higher (for example $102.4 \mathrm{kHz}$ at $1500 \mathrm{rpm}$ and a resolution of 4096 lines).

This example helps to view some limitation of the method. 


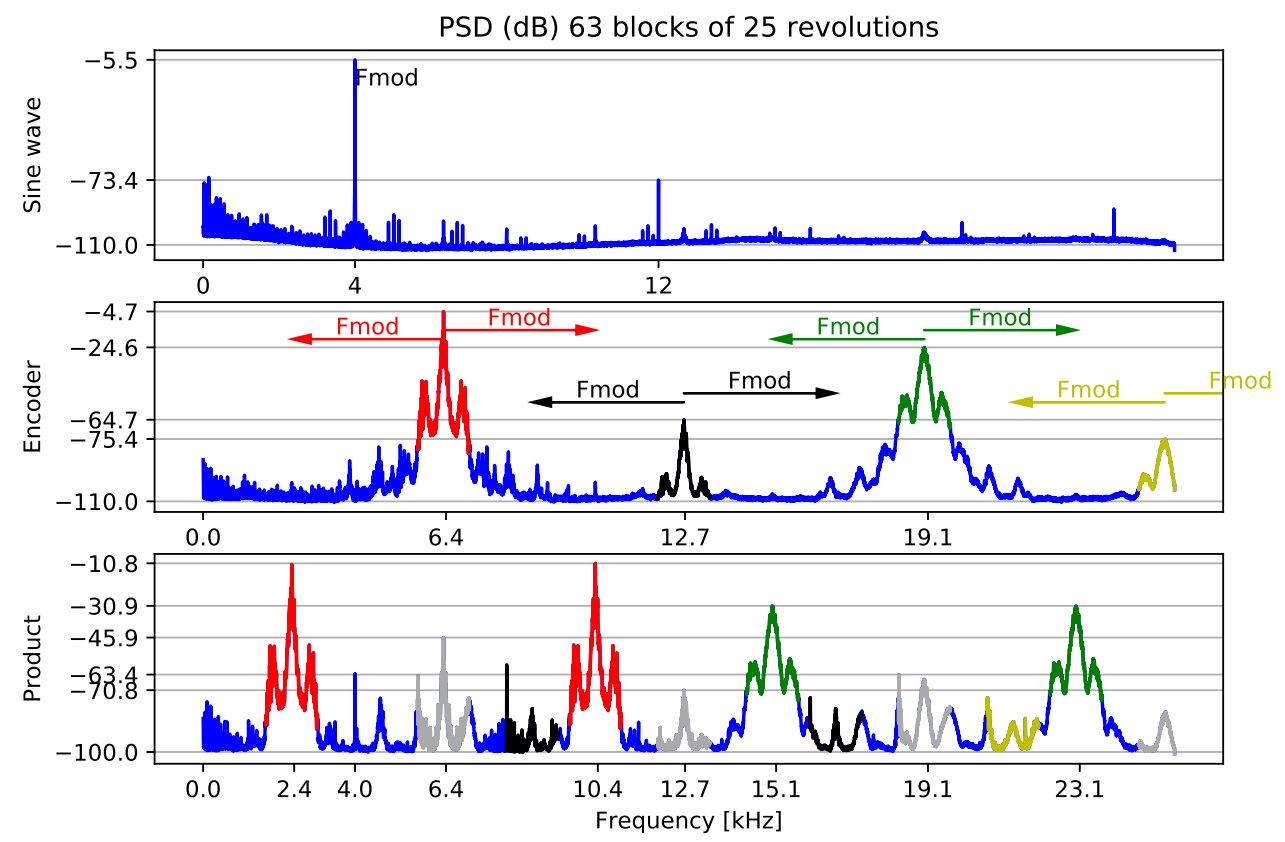

Figure 2: Illustration of High frequency Demodulation Technique.

\section{Limit : Frequency overlapping}

Let be $\beta$ the highest fluctuation frequency in $\mathrm{Hz}$ (like in the previous section). By looking at the bottom spectrum of figure 2 , it is possible to identify the following content :

- $\left[f_{\text {cod }}-f_{\text {mod }}-\beta, f_{\text {cod }}-f_{\text {mod }}+\beta\right]$ : left shifted encoder frequency used for instantaneous frequency estimation,

- $\left[k \cdot f_{\text {cod }} \pm f_{\text {mod }}-\beta, k \cdot f_{\text {cod }} \pm f_{\text {mod }}+\beta\right]$ : all left and right shifted encoder frequencies,

- $f_{\text {mod }}$ : modulation frequency (should not be here if $\varepsilon_{e}=0$ ),

- $\left[k \cdot f_{\text {cod }}-\beta, k \cdot f_{\text {cod }}+\beta\right]$ : encoder frequencies (should not be here if $\varepsilon_{s}=0$ ).

In order to have a good estimation, the left shifted encoder frequency should not overlap with the other area. Otherwise, the analytic signal will no longer be a sine wave and its phase will have a different meaning.

The frequency shifted red pattern should be kept in positive frequency. It leads to :

$$
\begin{aligned}
f_{\text {cod }}-f_{\text {mod }}-\beta & >0 \\
f_{\text {mod }} & <f_{\text {cod }}-\beta
\end{aligned}
$$

In order to access the first harmonic of the encoder, the demodulation frequency $f_{\text {mod }}$ is lower than $f_{\text {cod }}$.

If $\varepsilon_{e}$ is not 0 , no overlapping between the left frequency shifted red pattern and $f_{\text {mod }}$ implies that :

$$
\begin{aligned}
f_{\text {cod }}-f_{\text {mod }}+\beta & <f_{\text {mod }} \\
f_{\text {mod }} & >\frac{f_{\text {cod }}+\beta}{2} .
\end{aligned}
$$

If $\varepsilon_{s}$ is not 0 , no overlapping between the left red frequency and the original one implies that:

$$
\begin{aligned}
f_{\text {cod }}-f_{\text {mod }}+\beta & <f_{\text {cod }}-\beta \\
f_{\text {mod }} & >2 \beta
\end{aligned}
$$


If $\varepsilon_{s}=0$ and $\varepsilon_{e}=0$, no overlapping between frequency left and right shifted version implies that :

$$
\begin{aligned}
f_{\text {cod }}-f_{\text {mod }}+\beta & <f_{\text {cod }}+f_{\text {mod }}-\beta \\
f_{\text {mod }} & >\beta
\end{aligned}
$$

To conclude, the modulation frequency $f_{\text {mod }}$ should be lower than $f_{\text {cod }}-\beta$ and depending on whether $\varepsilon_{e}, \varepsilon_{s}$, or no $\varepsilon$ are take into account, $f_{\text {mod }}$ should be respectively greater than $\frac{f_{c o d}+\beta}{2}, 2 \beta$ or $\beta$.

Another way of thinking should be to find the demodulation frequency $f_{\text {modop }}$ that maximises the highest observable fluctuation frequency $\beta$ :

$$
\begin{gathered}
f_{\text {modop }}=\frac{2}{3} \cdot f_{\text {cod }} \quad, \quad \beta_{\text {max }}=\frac{1}{3} . f_{\text {cod }} \quad \text { considering } \varepsilon_{e} \text { and } \varepsilon_{s}, \\
f_{\text {modop }}=\frac{f_{\text {cod }}}{2} \quad, \quad \beta_{\text {max }}=\frac{f_{\text {cod }}}{2} \quad \text { if } \varepsilon_{e}=\varepsilon_{s}=0 .
\end{gathered}
$$

\begin{tabular}{|c|c|c|c|c|c|c|}
\hline Rotation frequency (rpm) & \multicolumn{4}{|c|}{1500} & 3000 & 6000 \\
\hline Optical encoder resolution & 256 & 512 & 1024 & 4096 & 4096 & 1024 \\
\hline Optical encoder mean frequency $(\mathrm{kHz})$ & 6.4 & 12.8 & 25.6 & 102.4 & 204.8 & 102.4 \\
\hline Optimal $f_{\text {modop }}$ frequency $(\mathrm{kHz})$ & 4.3 & 8.5 & 17.0 & 68.3 & 136.5 & 68.3 \\
\hline Theoretical maximum order & 85 & 170 & 341 & 1365 & 1365 & 341 \\
\hline
\end{tabular}

If offsets are not compensated, they limit $\beta_{\max }$ to order $N_{p p t} / 3$ instead of $N_{p p t} / 2$. The table 1 shows examples of demodulator setup.

Table 1: Example of demodulator setup

Since the sampling frequency of the acquisition device should be twice the $f_{\text {modop }}$ frequency in this particular case, this table is not compatible with a $51,2 \mathrm{kHz}$ maximum sampling frequency. To adapt it the constraint $f_{\text {mod }}>f_{\text {cod }}+\beta-f_{s} / 2$ should be added. This constraint leads to :

$$
f_{\text {modop }}=\max \left(\frac{2}{3} \cdot f_{\text {cod }}, f_{\text {cod }}-\frac{f_{s}}{4}\right) \quad, \quad \beta_{\text {max }}=\min \left(\frac{f_{\text {cod }}}{3}, \frac{f_{s}}{4}\right)
$$

or by dividing $\beta$ by $f_{\text {rot }}$ the maximum order $o_{\max }$ is :

\begin{tabular}{|c|c|c|c|c|c|c|}
\hline Rotation frequency (rpm) & \multicolumn{4}{|c|}{1500} & 3000 & 6000 \\
\hline Optical encoder resolution & 256 & 512 & 1024 & 4096 & 4096 & 1024 \\
\hline Optical encoder mean frequency $(\mathrm{kHz})$ & 6.4 & 12.8 & 25.6 & 102.4 & 204.8 & 102.4 \\
\hline Optimal $f_{\text {modop }}$ frequency $(\mathrm{kHz})$ & 4.3 & 8.5 & 17.0 & 89.6 & 192 & 89.6 \\
\hline Theoretical maximum order & 85 & 170 & 341 & 512 & 256 & 128 \\
\hline
\end{tabular}

$$
o_{\max }=\min \left(\frac{N_{p p t}}{3}, \frac{f_{s}}{4 \cdot f_{\text {rot }}}\right) .
$$

It means that the demodulation frequency $f_{\text {mod }}$ should be increased in order to shift more on the left the red pattern in figure 2, In this case, the needed sampling frequency become $f_{s}$.

Table 2: Example of demodulator setup to use not more than $f_{s}=51.2 \mathrm{kHz}$

These adjustments shows that the sampling frequency will limit the bandwidth in order. It should be kept in mind for comparison that theoretical order correspond to half of the equivalent optical encoder resolution. 


\section{Comparison with elapsed time}

In order to compare both methods, the full resolution optical encoder was recorded using elapsed time technique and high frequency demodulation technique. The frequency $f_{\text {mod }}$ is fixed to $90 \mathrm{kHz}$ according to table 2 .

The figure 3 compares two results for the two approaches. The instantaneous speed obtained with high frequency demodulation was resampled in angular domain and manually shifted in order to be compared to elapsed time speed. The band $\beta$ is chosen to be 3. $f_{\text {mesh }}$ (a lower band gives bad results). The comparison is valid only locally for the first graph. The blue curve corresponds to elapsed time, and the red curve to high frequency demodulation.

The two instantaneous speed curves are superposed. The high frequency method is smoother than elapsed time one due to quantification. The variation in the difference of $\pm 0.06 \mathrm{~Hz}$ is not so big be compared to the quantification step of $0.03 \mathrm{~Hz}$ for elapsed time method.

The spectrum is also close till order 30. After it seems that an additive noise is present in the high frequency demodulated signal : high peaks are still present but not smallest one. The filtering effect due to the band of 3. $f_{\text {mesh }}$ (order 69) becomes easily visible near order 100.

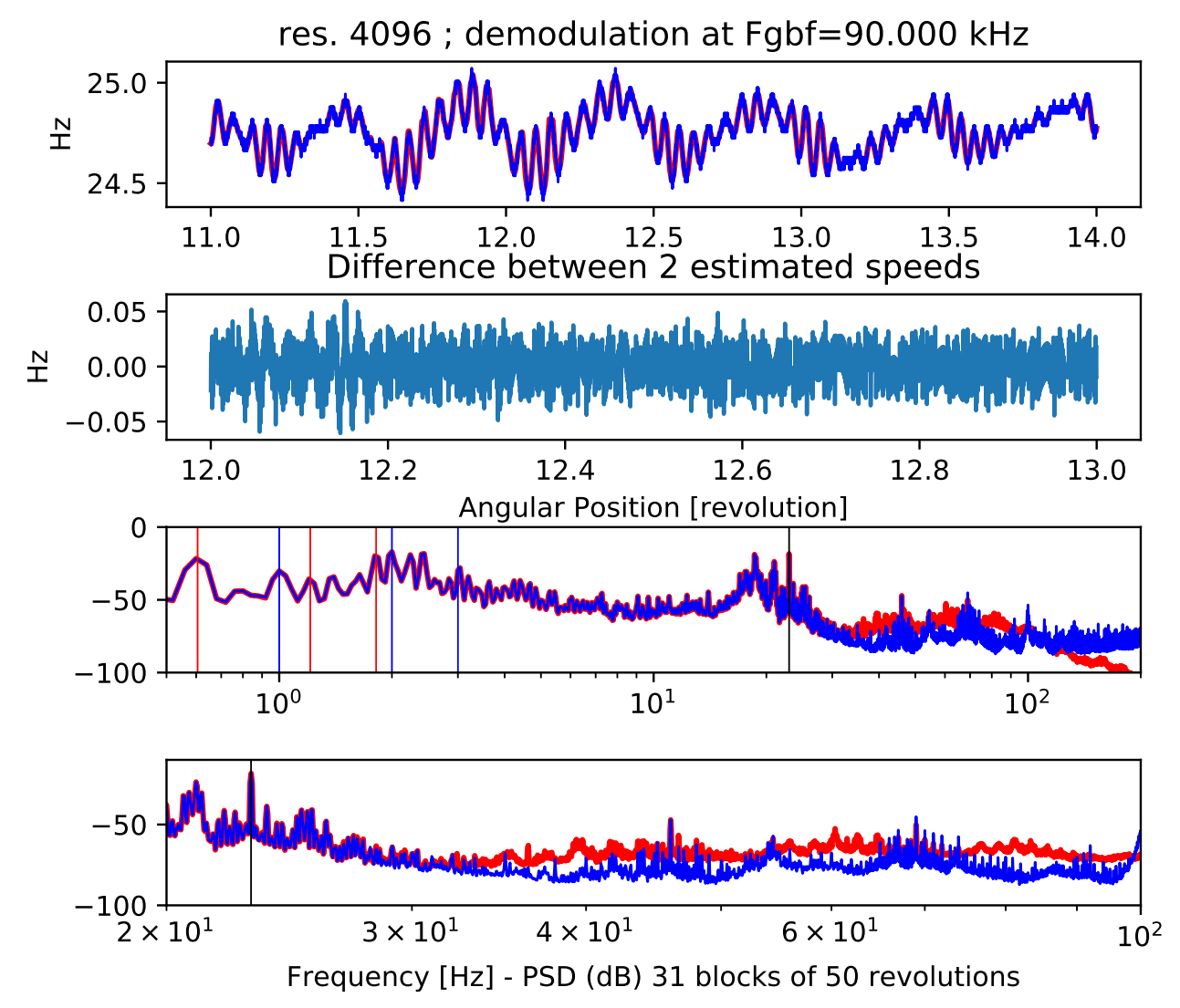

Figure 3: Comparison of Elapsed time and high frequency demodulation Technique.

\section{Conclusion}

We have proposed a high frequency demodulation technique that performs an analog demodulation prior to acquisition and enable to use the full optical encoder resolution. This technique known in telecommunication as super-heterodyne enables to inspect a restricted bandwidth in high frequency with a low sampling rate. The study of this new technique explains how to choose intermediary frequency for demodulation and some limitations due to the imperfection of electronic components. 
A test on a bench to compare this new method with elapsed time shows that the results are similar to the beginning of the spectrum and that an additive noise is present in high frequency. Therefore, this method seems to be interesting when no elapsed time system is present, or it is required to do simultaneous temporal sampling of other signal (acceleration, ...), or, if it is necessary to record very high frequency optical encoder signal.

\section{Acknowledgement}

The authors would like to thank Lucien Périsse that design this test bench for us.

\section{References}

[1] L. Renaudin, F. Bonnardot, O. Musy, J.B. Doray, and D. Rémond. Natural roller bearing fault detection by angular measurement of true instantaneous angular speed. Mechanical Systems and Signal Processing, 24(7):1998 - 2011, 2010. Special Issue: ISMA 2010.

[2] Ming Zhao, Xiaodong Jia, Jing Lin, Yaguo Lei, and Jay Lee. Instantaneous speed jitter detection via encoder signal and its application for the diagnosis of planetary gearbox. Mechanical Systems and Signal Processing, 98:16 - 31, 2018.

[3] H. André, F. Girardin, A. Bourdon, J. Antoni, and D. Rémond. Precision of the ias monitoring system based on the elapsed time method in the spectral domain. Mechanical Systems and Signal Processing, 44(1):14 - 30, 2014. Special Issue on Instantaneous Angular Speed (IAS) Processing and Angular Applications.

[4] K. Lizoul, H. André, and F. Guillet. Improvement of instantaneous angular speed estimation based on frequency demodulation method under non stationary conditions. In Proceedings of ISMA2018 and USD2018, Leuven, 17-19 September 2018.

[5] F. Bonnardot and J. Al Fallah. Non-uniform (re)sampling for optical encoder. In First World Congress on Condition Monitoring (WCCM), London, 13-16 June 2017.

[6] M. El Badaoui and F. Bonnardot. Impact of the non-uniform angular sampling on mechanical signals. Mechanical Systems and Signal Processing, 44(1-2):199-210, February 2014.

[7] Heidenhain. Rotary Encoders brochure, 2018.

[8] F. Bonnardot, M. El Badaoui, R.B. Randall, J. Danière, and F. Guillet. Use of the acceleration signal of a gearbox in order to perform angular resampling (with limited speed fluctuation). Mechanical Systems and Signal Processing, 19(4):766 - 785, 2005. 